\title{
Fuzzy Logic Based Control System for Intelligent Washing Machines
}

\author{
Abdalla Fadel ${ }^{1}$, Mohamed Mhereeg ${ }^{2}$,Mona Shlibek ${ }^{3}$ and Manal Shlibek ${ }^{4}$ \\ ${ }^{1}$ Professor - School of Engineering and Applied Science/ Libyan Academy. \\ ${ }^{2}$ Assistant Professor- Faculty of Information Technology- Tripoli University. \\ ${ }^{3}$ Engineer at GECOL- MSc- Electrical Engineering / Tripoli University. \\ ${ }^{4}$ Engineer at GECOL- MSc- Electrical Engineering/ Libyan Academy.
}

\begin{abstract}
ARTICLE INFO
Keywords:

Mamdani, Surface

Viewer, Wash Time,

Spin Time, and Rinse

Time.
\end{abstract}

\begin{abstract}
Nowadays, in the Libyan households, washing machines are a very common items. It is not only a big time saver over hand washing, but also it saves a great effort, people can carry out another task and then return back to the machine to get their clothes clean and even dry. The only things they need to do are load the clothes into the machine, throw detergent in, run it, and walk away. This paper shows the importance of fuzzy logic control based washing machine to get a suitable wash, rinse, and spin time for different types and amount of clothes and amount of dirtiness. The process is based on fuzzy inference system. The signals that come out from sensors are fed to the fuzzy controller as non-precise inputs to get the desired output (a crisp value of washing time, rinsing time and spinning time). The Simulation was done by MATLAB's fuzzy logic toolbox.
\end{abstract}

\section{Introduction}

Fuzzy logic controller has many practical advantages over the other controllers, not only because of its simplicity, effectively, and flexibility, but also it can handle problems with imprecise and incomplete data, giving us a desired data with very accurate and precise results corresponding on a wide range of operating conditions expressed in linguistic terms. As there are many types of fuzzy logic controller, in this paper the most commonly used fuzzy inference system is the so called Mamdani method. In 1974, Professor Ebrahim Mamdani of London University built one of the first fuzzy systems to control a steam engine and boiler combination [1]. The Mamdani fuzzy inference system is designed in four steps: Fuzzification of the input variables, Rule evaluation; Aggregation of the rule outputs, and finally Defuzzification [2]. Alhanjouri and

\footnotetext{
$\square$ Corresponding Author E-Mail Address: manal.shlibek@gmail.com
} 
Alhaddad's optimize wash time using fuzzy logic [3]. They suggested that dirt type and degree of dirtiness are inputs of fuzzy controller, whereas the output of fuzzy controller is a wash time. On the other hand, inputs of fuzzy logic controller including, type of dirt, dirtiness of clothes were selected in by researcher Agarwal provided in his proposed system 9 rules for the FLC [4]. Kumar and Haider, in 2013, proposed in order to decrease washing time as a response of fuzzy controller, the quantity of clothes and dirtiness were chosen as input [5]. Rao Farhat was interested in a washing machine with two inputs (Saturation Time and Dirtiness) and one output (Wash Time). For practical purposes type and amount of detergent has been kept manual [6]. Whereas, in 2014, Akram, Habib and Javed used to obtain wash time as a crisp output from a fuzzy input which are type of dirt and degree of dirt [7]. On the other hand, during this year '2014', Demetgul, Ulkir, Waqar found to get best results it is necessary to consider four input parameters of fuzzy logic including: Amount of Dirt, Type of Dirt, Sensitivity of Cloth, Amount of Cloths, and four outputs parameters including: Washing Time, Washing Speed, Amount of Detergent, Amount of Water and Water Hotness[8]. While, in 2015, Hatagar and Halase objected to save lot of time, electricity and water for washing the cloth. So they considered type-of-dirt and dirtiness of clothes as inputs to fuzzy controller [9]. In 2016, Agarwal, Mishra, and Dixit introduce five input variables including Type of Dirt, Turbidity of Cloth, Mass of Cloth, Sensitivity of Cloth, Water Hardness and five output variables including Wash Time, Wash Speed, Amount of Water, Amount of Detergent, Water Hotness[10]. Anita , and Bhawna Hooda in same year, 2016, have proposed the design of fuzzy logic controller which is having five inputs to give an exact wash, rinse and spin time of fully automatic machine. The objective of this is only to save a lot of time, electricity, detergent and water for washing the cloth. The proposed FLC is simulated using Fuzzy Logic Toolbox of MATLAB. The result of this report is used to calculate the wash time, rinse time and spin time for different type of input conditions [11]. Washing machines are commonly used household appliances. The problem of designing washing machines is how much length of wash time, rinse time and spin time should be based on the different clothes. That's why this study is interesting because it illustrates the process that can be used to get a appropriate washing, rinsing, and spinning time for different types and amount of cloths. So, the paper will cover the characteristic of designing and developing of Fuzzy Logic based washing machine. Such a machine makes use of the Fuzzy Logic mechanism to smartly calculate the amount of washing, rinsing, and spinning time in order to provide a precise real-time for the machine motor. The paper has three different outputs "which are wash time, rinse time and spin time" resultant from three different inputs "which are type of clothes, amount of clothes and amount of dirtiness". These inputs and outputs were controlled by fuzzy logic controller. Depending on the types and amount of clothes and amount of dirtiness of cloths, washing machine will automatically adjust its washing, rinsing, spinning time using Mamdani controller. Consequently, Fuzzy logic control system can be used to calculate the processing time of washing machine for different cloths.

\section{Proposed Design for FLC of Smart Washing Machine}

\subsection{General Idea of Fuzzy Control System For Washing Machine}

This work is a control system that provides a solution of run- time optimization problem by placing the system which will be constructed utilizing the Fuzzy Logic Controller. In general when we use washing machine, we load the clothes into the machine, throw detergent in, select the duration of washing time based on type and amount of clothes and type of dirtiness or greasy and run it. Once switch the washing machine on, sensors sense these parameters which are considered in this 
paper as inputs that will be fed to the fuzzy controller to get the exact outputs which are considered in this paper as wash time, rinse and spin time. For getting correct wash time simply, more accurately and precisely, we use fuzzy logic instead of formulating a mathematical model. As it is very difficult to find out mathematically the relationship between amount of cloths, dirt and the length of washing time required.

\subsection{The Designed System}

The fuzzy inference system or FIS, is designed in four steps: first step is fuzzification of the input variables. In this stage, crisp inputs are converted into fuzzy inputs. So, membership functions are created to represent each crisp input depending on linguistic terms and their ranges. Second step is rule evaluation to evaluate the process; Third step is Aggregation of the rule outputs, and finally the fourth step is defuzzification which is the process when the fuzzy inputs are changed into crisp outputs. In this project we use Mamdani style and defuzzification method is centroid. The proposed Fuzzy Logic Controller for washing machine consists of three Linguistic inputs LIs.

\section{- $\quad$ 2.2.1 Input Parameters:}

1) Type of clothes (TC)

2) Amount of clothes (AC)

3) Amount of dirtiness (AD)

These three inputs are fed to the fuzzy controller to obtain the next three outputs as below.

\section{- $\quad$ 2.2.2 Output Parameters:}

1) Wash time (Wash)

2) Rinse time (Rinse)

3) Spin time (Spin)

\section{Fuzzy Logic Modelling of Washing Machine}

In this paper, the control logic was used to checks the system continuously and execute the operation perfectly. The actuation of the Mamdani controller is giving calculations of running time with high accurate.

\section{- $\quad$ 3.1 The Membership Function for Fuzzy Inputs and Fuzzy Outputs}

After commencing the proposed system that include three input and three output variables as mentioned above, now we find out membership functions for each variable for the proposed fuzzy system and their corresponding fuzzy memberships. The washing machine fuzzy inference system is shown in figure 1.

Figure1. FIS Editor ToolBox/MATLAB

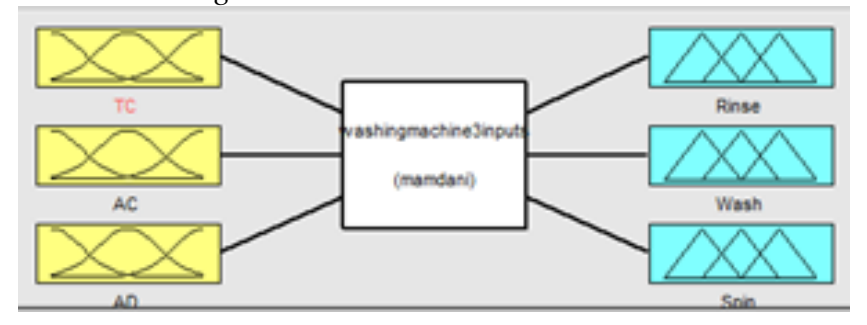


In this Fuzzy Logic Modeling of Washing Machine, linguistic terms and their ranges of fuzzy inputs and fuzzy outputs are illustrated as below:

\section{- $\quad$ 3.1.1 Fuzzy Inputs}

In fuzzy logic, crisp inputs are converted into fuzzy inputs. So, membership functions are created to represent each crisp input depending on linguistic terms and their ranges. In this paper, the parameters are used to define membership function of Type of clothes (TC) thin, thick, and jeans are [-50 0 50], [2 50 80] and [50 100 150] respectively. Similarly Membership function for Amount of clothes (AC) little, normal, and large are [ $\left.\begin{array}{lll}-5 & 0 & 5\end{array}\right],\left[\begin{array}{lll}2 & 5 & 8\end{array}\right]$, and [ [ 10 10 15$]$ correspondingly. Also, Membership function for Amount of dirtiness (AD) small [ [-50 0 50], normal [20 50 80], and large [50 100 150]. The input membership functions of fuzzy logic shown in figures $2,3,4$.

Figure2. Membership Function of Type of Clothes

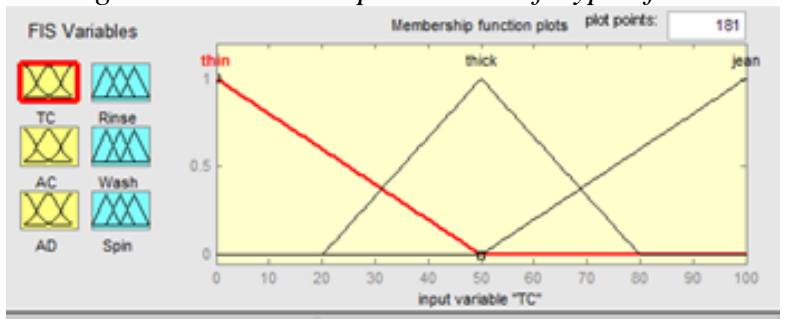

Figure3. Membership Function of Amount of Clothes

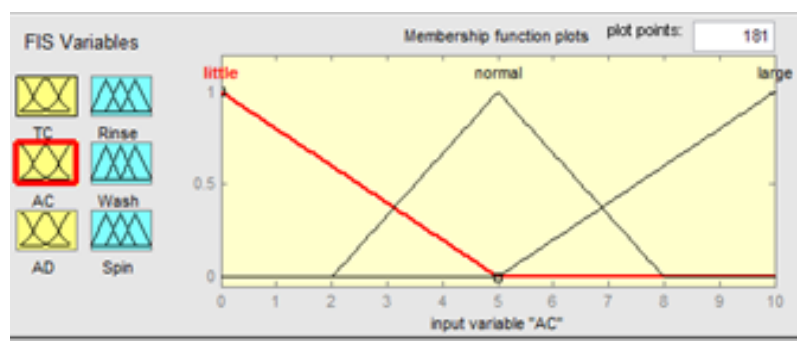

Figure.4. Membership Function of Amount of Dirtiness
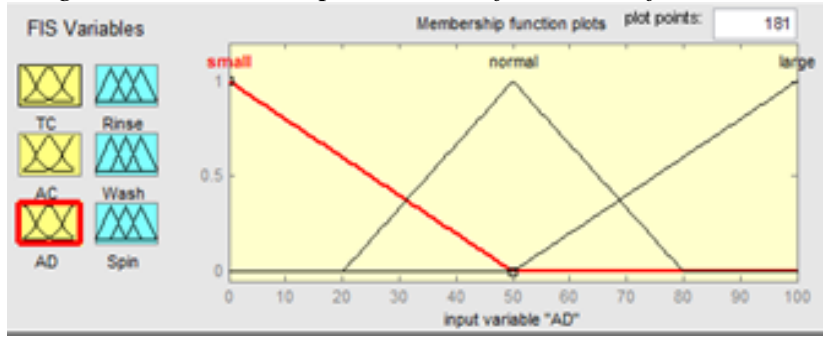

- $\quad$ 3.1.2 Fuzzy Outputs

MF for Rinse time very small, small, normal, long, and very long are [-12.5 0 12.5], [0 12.5 25], [15 25 35], [25 35 45], [40 60 80]. MF for washing time small, normal and large are [-20 0 20],[10 
25 40] and [35 50 50].The membership functions of Spin time very small, small, normal, large and very large [0 0 40], [30 52.5 75], [50 75 100], [75 107.5 140], [120 180 480] respectively. The output membership functions of fuzzy logic shown in figures 5, 6, 7 .

Figure. 5. Membership Function of Rinse Time

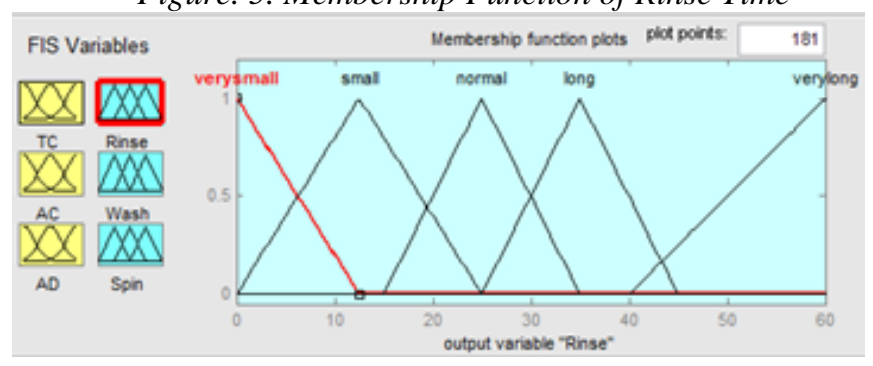

Figure. 6. Membership Function of Wash Time

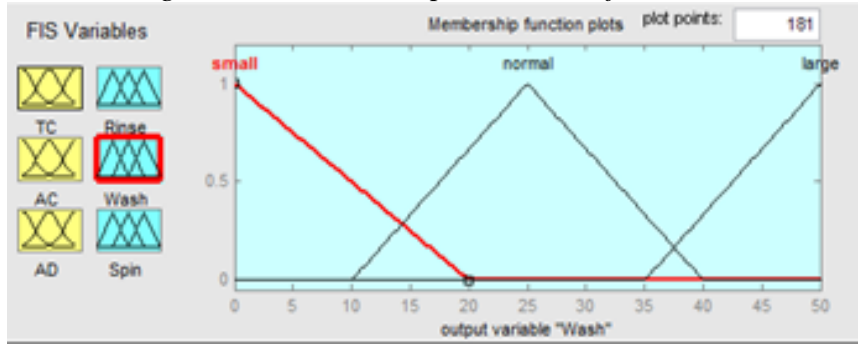

Figure. 7. Membership Function of Spin Time

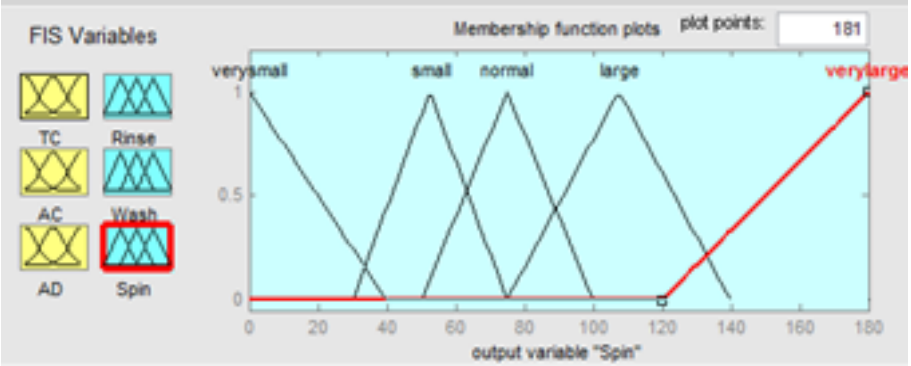

- $\quad$ 3.2 The Mamdani-style fuzzy inference process is performed in four steps

The type of controller used in this model is "Mamdani". The Aggregation is Max. The Implication is Min. The membership functions are triangular, and they are symmetrical.

\section{- 3.2.1 Fuzzification Method}

Fuzzification of input variable is the process when crisp values are changed into fuzzy values. Fuzziness assists us to evaluate the rules.

\section{- 3.2.2 Control Rules}

The evaluation rules in fuzzy design for washing machine are shown in figures (8.a, 8.b, 8.c) in order to derive the output. The system will operate under these rules. As a result, the decisions will be made which represents a controller's response. The fuzzy rules are either the sets of ( If A Then B ) statements, or the sets of ( If A is X AND B is Y Then C is Z) statements, or the sets of of ( If $\mathrm{A}$ is $\mathrm{X}$ OR B is $\mathrm{Y}$ Then $\mathrm{C}$ is $\mathrm{Z}$ ) statements. 
Figure 8.a, Rule Editor from ToolBox/ MATLAB

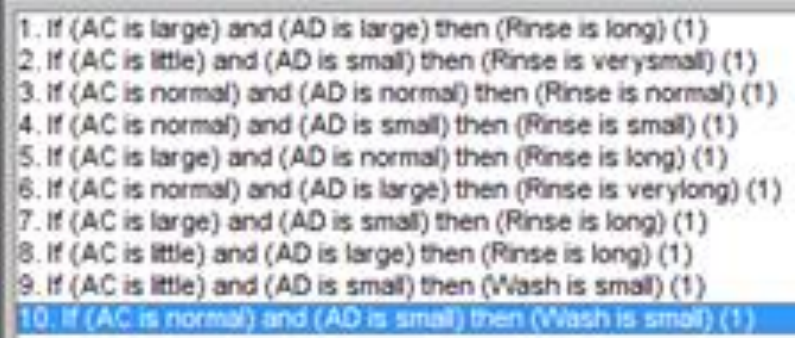

Figure 8.b, Rule Editor from ToolBox/ MATLAB

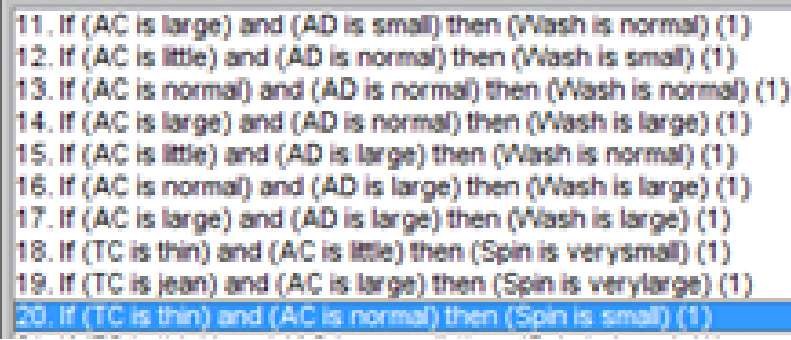

Figure 8.c, Rule Editor from ToolBox/ MATLAB

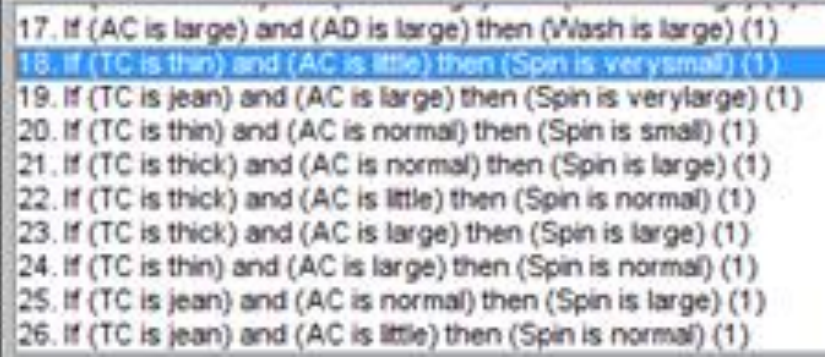

- 3.2.3 Aggregation of the rule outputs

Aggregation is the process of unification of the outputs of all rules which are extracted from membership functions. In order to get only a single fuzzy set, all rules are aggregated to combine them into a particular fuzzy set. The aggregated output fuzzy set is the input for defuzzification process.

\section{- $\quad$ 3.2.4 Defuzzification}

The fuzzy set that comes out from the aggregation process must be a crisp number. So, the conversion process from a fuzzy set to a crisp set is called defuzzification. So, a single number is the output of defuzzification process which is in turns the controller's response. As there are quite a lot of defuzzification methods, in this paper, we used the most common one which is Centroid method.

\section{- 4. Results Toolbox/MATLAB}

As it is known, control rules are established by applying Min-Max operator. These rules are pointed up in the form of 3D graphs in next figures. The figure 9. shows the relationship between input and output parameters. 
Figure 9. Rule Viewer from Fuzzy Logic Toolbox/ MATLAB

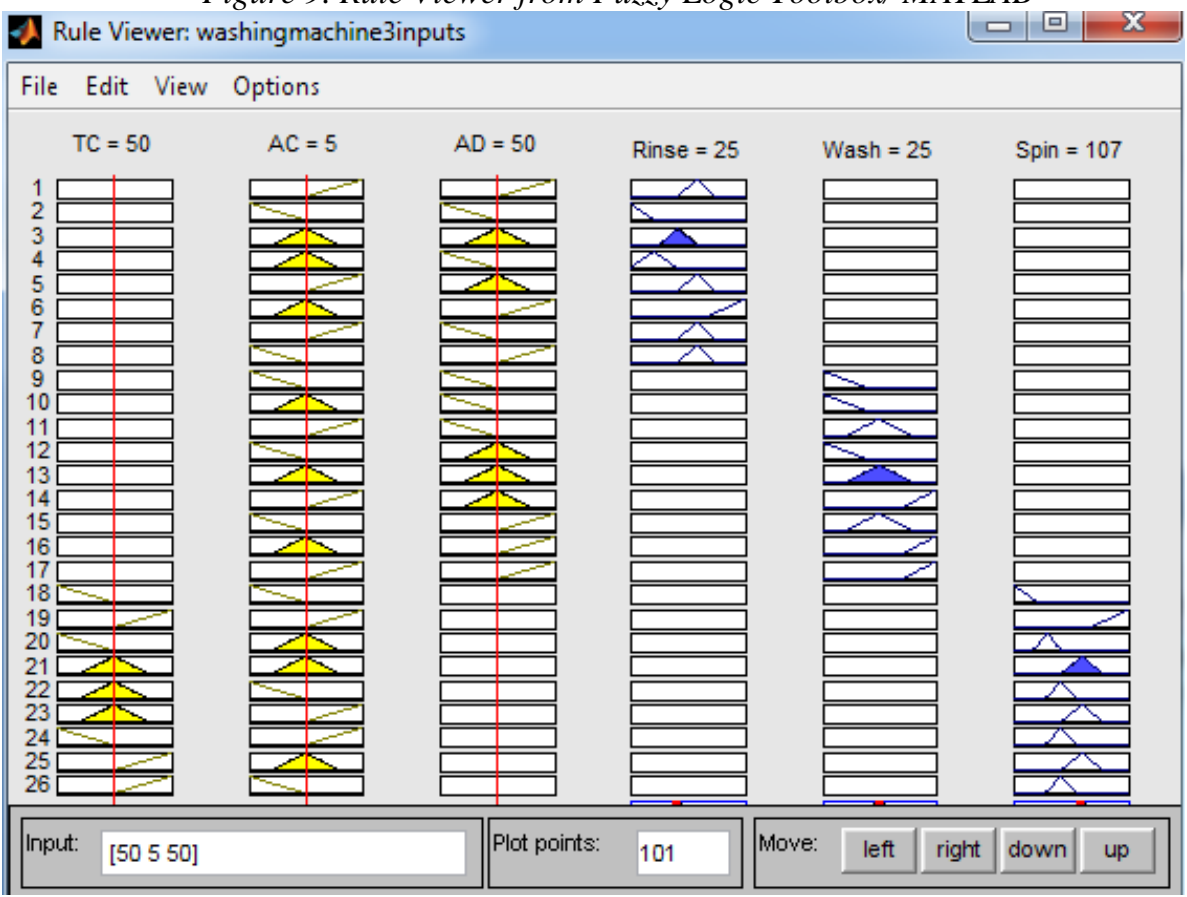

\section{Surface Viewer}

By using MATLAB's fuzzy logic toolbox the fuzzy inference system have been created. Consequently, The surface rule views of the fuzzy logic modeling rules for washing machine are shown in figures (10.a, 10.b, 11.a, 11.b, and 12.a, 12.b) as 3D graphs. Each surface view is a response surface of the input output relations.

Figure10.a, Surface Viewer from Fuzzy Logic Toolbox

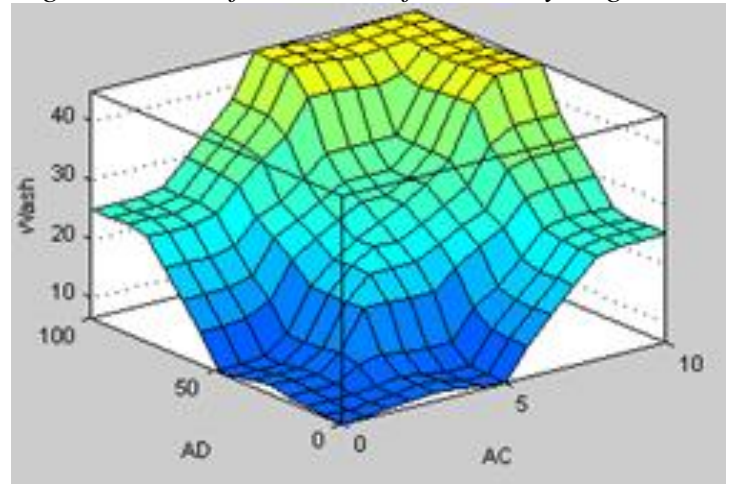


Figure10.b, Surface Viewer from Fuzzy Logic Toolbox

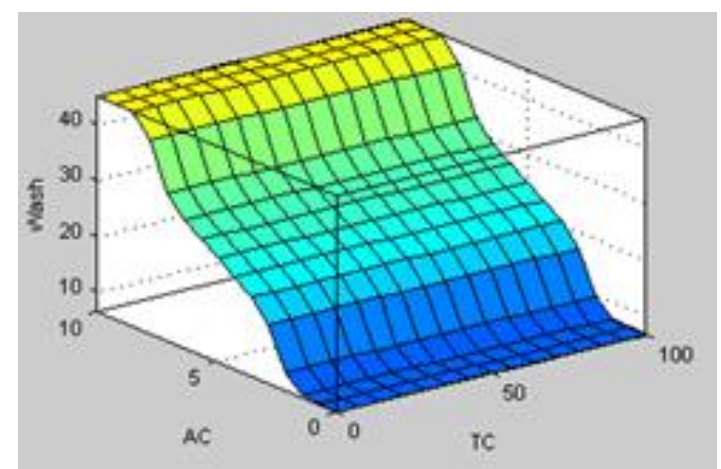

It can be clearly seen from figure 10.a, washing time isn't affected much by the amount of dirtiness. While, from figure 10.b, that the amount of clothes has a much higher affect in washing time than the type of clothes.

Figure11.a, Surface Viewer from Fuzzy Logic Toolbox

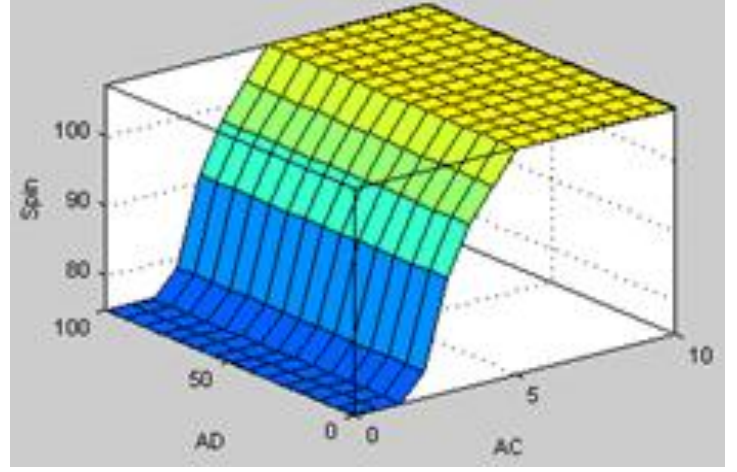

Figure11.b, Surface Viewer from Fuzzy Logic Toolbox

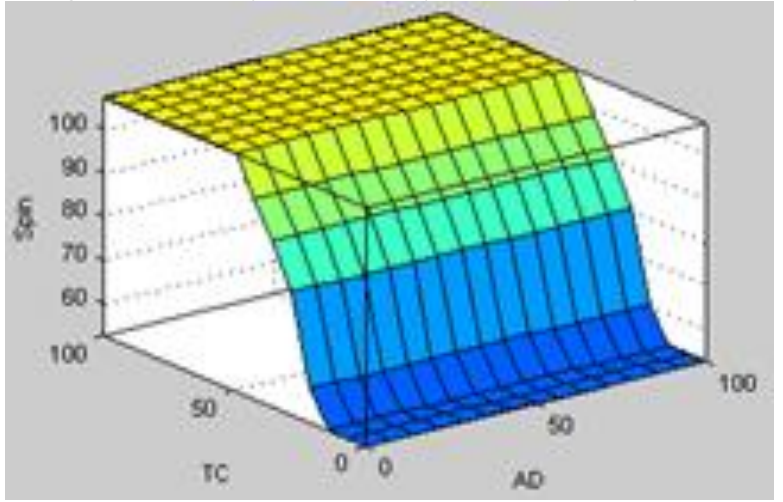

However, figures 11.a and 11.b present the Spin time is very much affected by both the amount and the type of clothes. The amount of dirtiness has no effect at all on Spin time. 
Figure12.a, Surface Viewer from Fuzzy Logic Toolbox

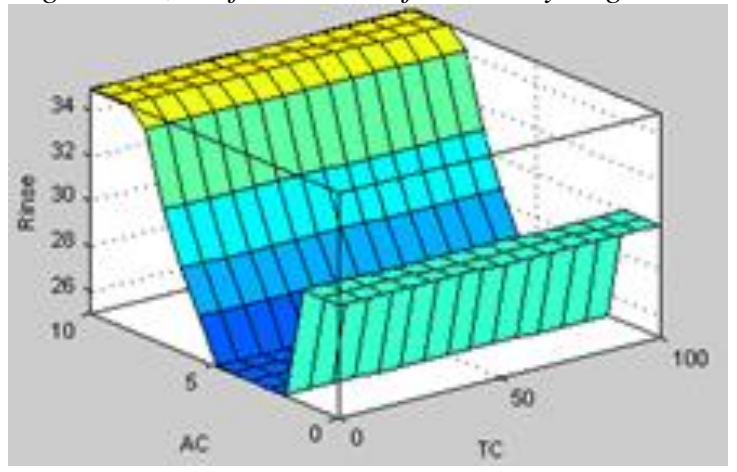

Figure12.b, Surface Viewer from Fuzzy Logic Toolbox

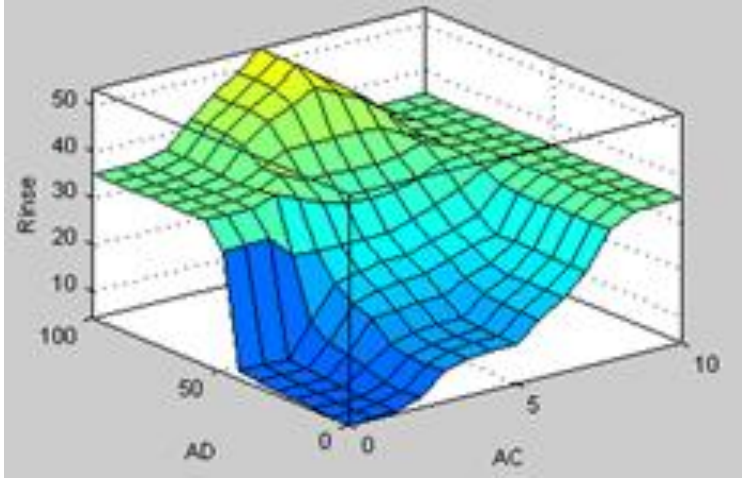

Figures 12.a and 12.b show the amount of both dirt and clothes are the most important factors which regulate the rinsing time. Whereas, the amount of clothes is directly proportional to the rinse time compared with amount of dirtiness. Figures (13.a, 13.b, 13.c) show the way the machine will response in different conditions. As sensors sense the input values and using the proposed system , the response of sensors are fuzzyfied and then by using if-else rules and aggregation, and lastly the output values are extracted from defuzzification method.

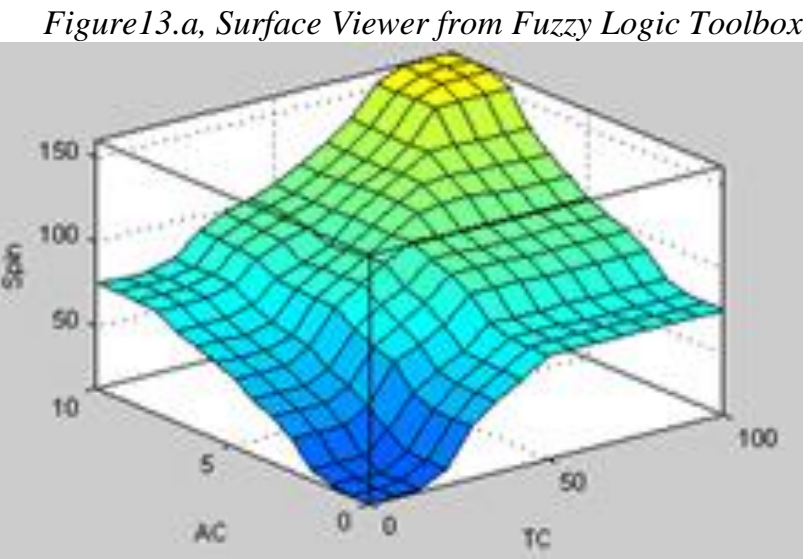


Figure13.b, Surface Viewer from Fuzzy Logic Toolbox

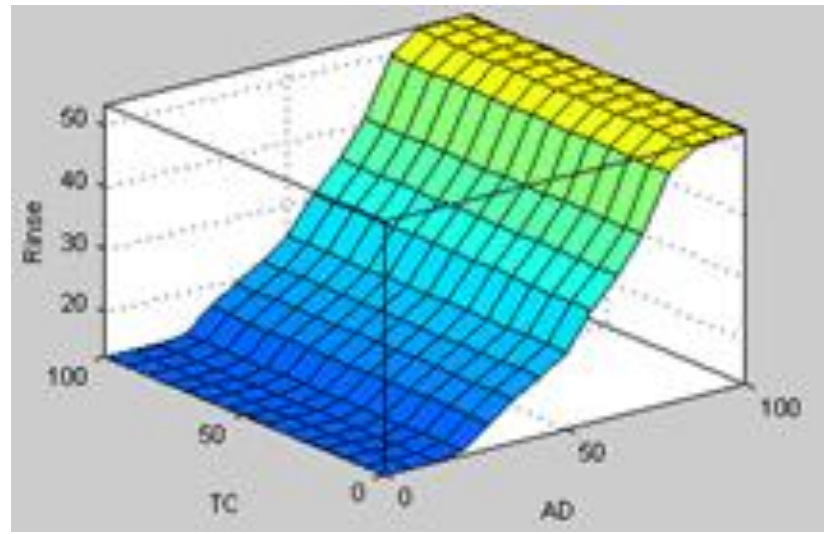

Figure13.c, Surface Viewer from Fuzzy Logic Toolbox

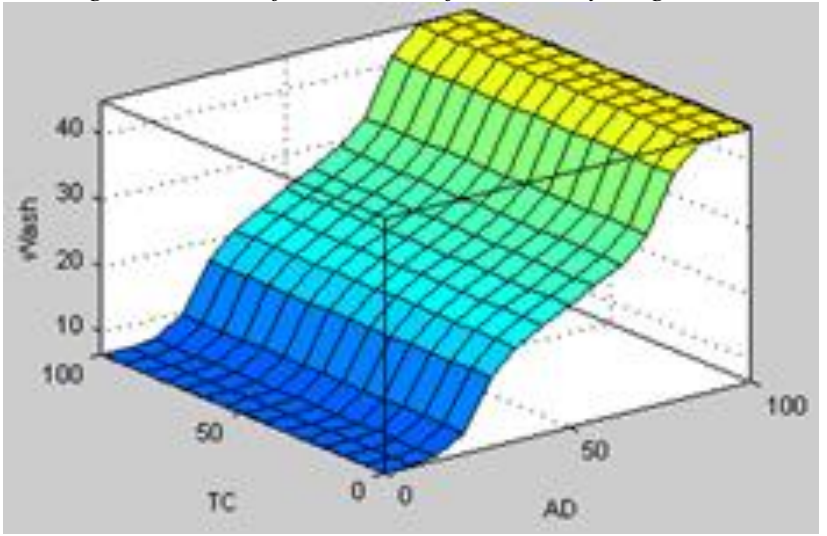

\section{Conclusion}

This paper illustrates fuzzy inference system using Mamdani controller type. From the use of fuzzy logic control, the machine will response in different conditions. This leads to know exactly how much time does the machine needs to finish it's work with best result and more saving consumed power. The conventional washing machines require the human interaction to make a decision about the amount of wash, spin and even rinse time do we need for different type and amount of clothes corresponding to amount of dirt. This makes the washing machine more intelligent and accurate.

\section{References}

Mamdani, E.H., 1974, “Application of Fuzzy Algorithms for Control of Simple Dynamic Plant”, Proc. IEEE, 121(12), 1585-1588.

Bldg 326, DK-2800 Lyngby, DENMARK, September 30, 1999, Technical University of

Denmark, Department of Automation,. Tech. report no 98-E 864 (design). 
Mohammed Alhanjouri, and Ahmed A. Alhaddad, OPTIMIZE WASH TIME OF WASHING MACHINE USING FUZZY LOGIC Associate Professor, Computer Engineering Department, Islamic University of Gaza E-mail: mhanjouri@iugaza.edu.ps, Postgraduate Student, Electrical Engineering Department, Islamic University of Gaza E-mail: aah4190@hotmail.com.

Manish Aggarwal, 2011, Fuzzy Logic Controller for Washing Machine, IIT Kharagpur.

Deepak Kumar, Yousuf Haider, Fuzzy Logic Based Control System for Washing Machines, Dept. of Electrical Engineering, NITTTR Chandigarh, Dept. of CSE, NITTTR Chandigarh, Panjab University, India.

Rao Farhat Masood, APPLICATIONOF FUZZY LOGIC IN DESIGN OF SMART WASHING MACHINE/ / National University of Sciences and Technology, Pakistan.

Muhammad Akram, Shaista Habib and Imran Javed, Intuitionistic Fuzzy Logic Control for Washing Machines Punjab University College of Information Technology, University of the Punjab, Old Campus, Lahore-54000, Pakistan.

Mustafa Demetgul, Osman Ulkir, Tayyab Waqar, No. 3, 2014, Washing Machine Using Fuzzy Logic. Automation, Control and Intelligent Systems. Vol. 2, pp. 27-32. doi: 10.11648/j.acis.20140203.11.

Sudha Hatagar, S.V. Halase, Three Input -One Output Fuzzy logic control of Washing Machine Dept. of Physics, Karnataka State Women's University, Jnana Shakti campus, Bijapur-586108, India, Registrar (Evaluation), Gulbarga University, Jnana Ganga campus, Gulbarga-585106, India.

Ankur Agarwal M., and Mukesh Dixit, Design of an Improved Fuzzy Logic based Control System for Washing Machines, Tech Scholar Department of Computer Science and Engineering Radharaman Engineering College, Bhopal (M.P.). Ankur Mishra Assistant Professor Department of Computer Science and Engineering Radharaman Engineering College, Bhopal (M.P.). Mukesh Dixit Head of Department Department of Computer Science and Engineering Radharaman Engineering College, Bhopal (M.P.).

3. Rohtak, Haryana, INDIA. Bhawna Hooda, Performance Analysis of Fuzzy Based Washing Machine Anita, Electronics \& Communication Engineering, R.N College of Engineering and Management, Rohtak, Haryana, INDIA. Bhawna Hooda, HOD, Electronics \& Communication Engineering, R.N College of Engineering and Management, Rohtak, Haryana, INDIA. 